\title{
FEBRERO DE 1976
}

1'r AÑo - Núm. 2
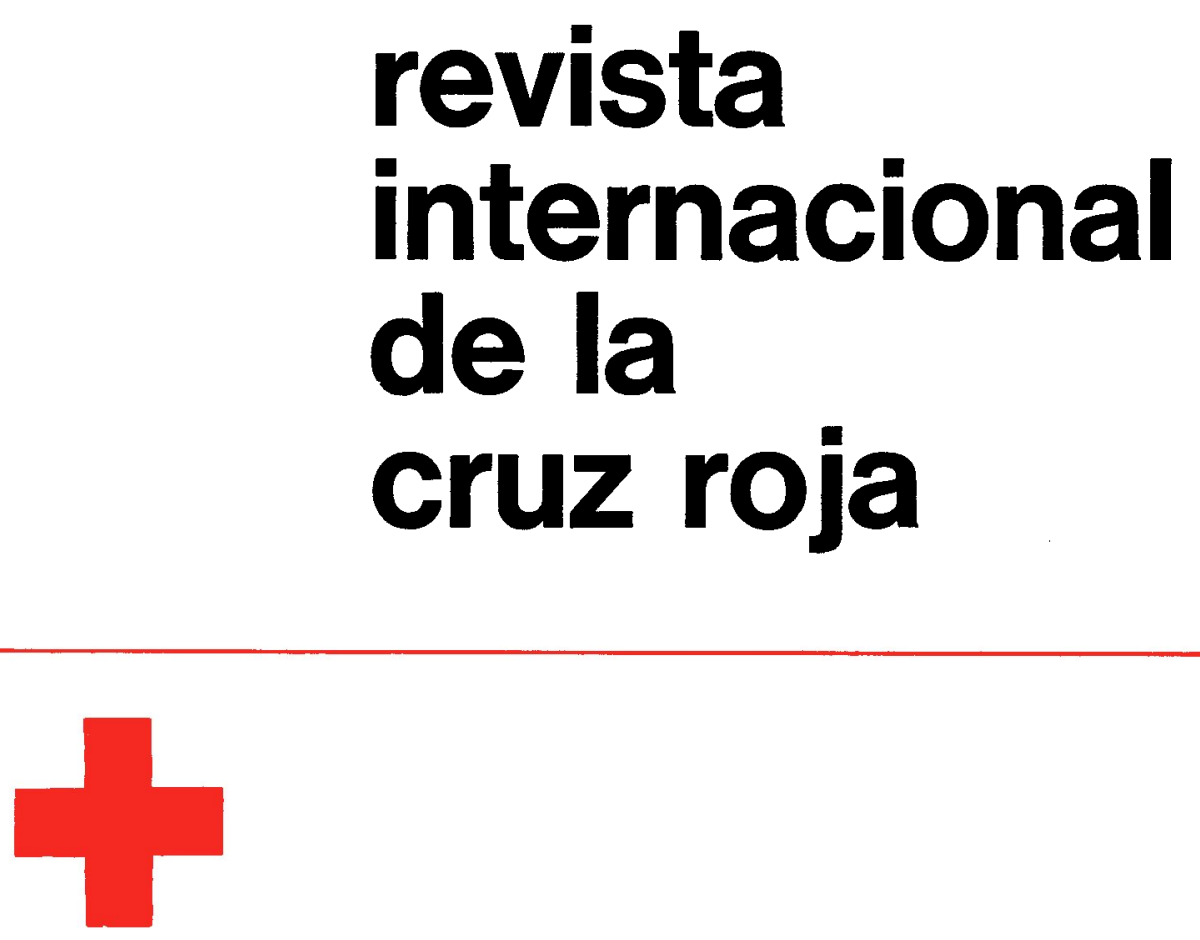

INTER ARMA CARITAS

GINEBRA

COMITÉ INTERNACIONAL DE LA CRUZ ROJA FUNDADO EN 1863 


\title{
COMITÉ INTERNACIONAL DE LA CRUZ ROJA
}

Sr. ERIC MARTIN, doctor en medicina, profesor honorario de la Universidad, Ginebra, presidente (miembro desde noviembre de 1973)

Sr. JEAN PICTET, doctor en derecho, presidente de la Comisión Jurídica, director del Instituto Henry Dunant, profesor asociado de la Universidad de Ginebra, vicepresidente (1967)

Sr. HARALD HUBER, doctor en derecho, juez federal, vicepresidente (1969)

Sra. DENISE BINDSCHEDLER-ROBERT, doctora en derecho, profesora del Instituto Universitario de Altos Estudios Internacionales de Ginebra, jue $z$ en el Tribunal Europeo de Derechos del Hombre (1967)

Sr. MARCEL A. NAVILLE, licenciado en letras, ex presidente del CICR (de 1969 a 1973)

Sr. JACQUES F. DE ROUGEMONT, doctor en medicina (1967)

Sr. ROGER GALLOPIN, doctor en derecho, ex director general del CICR (1967)

Sr. WALDEMAR JUCKER, doctor en derecho, secretario de la Unión Sindical Suiza (1967)

Sr. VICTOR H. UMBRICHT, doctor en derecho, administrador (1970)

Sr. PIERRE MICHELI, licenciado en derecho, ex embajador (1971)

Sr. GILBERT ÉTIENNE, profesor del Instituto Universitario de Altos Estudios Internacionales y del Instituto de Estudios de Desarrollo, Ginebra (1973)

Sr. ULRICH MIDDENDORP, doctor en medicina, jefe de la clínica quirúrgica del Hospital cantonal, Winterthur (1973)

Sra. MARION BOVEE-ROTHENBACH, diplomada M.S.W. de la Universidad de Michigan, profesor asistente de la Escuela de Ciencias Sociales y Políticas de la Universidad, Lausana (1973)

Sr. HANS PETER TSCHUDI, doctor en derecho, ex consejero federal (1973)

Sr. HENRY HUGUENIN, director de banco (1974)

Sr. GOTTFRIED DE SMIT, administrador (1974)

Sr. JAKOB BURCKHARDT, doctor en derecho, ministro plenipotenciario, presidente del Consejo de las Escuelas Politécnicas Federales (1975)

Sr. THOMAS FLEINER, doctor en derecho, profesor de la Universidad de Friburgo (1975)

Sr. ALEXANDRE HAY, abogado, director general del Banco Nacional Suizo, presidente electo (1975)

Sr. HERBERT LÜTHY, doctor en filosofía, profesor de historia en la Universidad de Basilea (1975)

Miembros honorarios: Sr. JACQUES CHENEVIËRE, vicepresidente honorario; Srta. LUCIE ODIER, vicepresidenta honoraria; Sres. HANS BACHMANN, GUILLAUME BORDIER, PAUL CARRY, Sra. MARGUERITE GAUTIER-VAN BERCHEM, Sres. ADOLPHE GRAEDEL, ÉDOUARD DE HALLER, RODOLFO OLGIATI, MAX PETITPIERRE, PAUL RUEGGER, DIETRICH SCHINDLER, FRÉDÉRIC SIORDET, ALFREDO VANNOTTI.

\section{CONSEJO EJECUTIVO}

\author{
Sr. ROGER GALLOPIN, presidente \\ Sr. VICTOR H. UMBRICHT, vicepresidente \\ Sra. DENISE BINDSCHEDLER-ROBERT \\ Sr. GILBERT ETIENNE \\ Dr. ULRICH MIDDENDORP \\ Sr. JEAN PICTET \\ Sr. GOTTFRIED DE SMIT \\ Sr. PIERRE MICHELI, miembro suplente
}




\section{REVISTA INTERNACIONAL DE LA CRUZ ROJA}

\section{ÍNDICE}

COMITÉ INTERNACIONAL DE LA CRUZ ROJA

EN EL MUNDO

DE LA CRUZ ROJA

HECHOS Y DOCUMENTOS
FEBRERO DE 1976 - Núm. 2

Yolanda Diallo: Derecho humanitario y derecho tradicional africano . . . . . . . . . . . . . 55

Conferencia de expertos gubernamental sobre las armas; segunda reunión . . . . . . . . . . 62

\section{En Ginebra:}

Miembro honorario del CICR . . . . . . . . 65

Puntualizaciones del CICR . . . . . . . . . . . 66

Participación en los Convenios de Ginebra . . . 67

Una nueva publicación . . . . . . . . . . . 67

Testimonio de generosidad: El Fondo Francés Maurice de Madre . . . . . . . . . . . . 68

Un nuevo índice de la Revista Internacional de la Cruz Roja: 1962-1974 . . . . . . . . . . . 70

Publicaciones del CICR desde 1966 hasta 1975 . 72

Asistencia en favor de las víctimas saharauis . . 83

Mauritania . . . . . . . . . . . . . . . 84

Libia . . . . . . . . . . . . . . 87

República Democrática Alemana . . . . . . . 89

República de Corea . . . . . . . . . . . . . 90

Día Mundial de la Cruz Roja. . . . . . . . . 90

Japón . . . . . . . . . . . . . . . . . . . 92

Repatriaciones organizadas por la Cruz Roja Internacional . . . . . . . . . . . . . . 95

Una resolución sobre las armas químicas y biológicas . . . . . . . . . . . . . . . . . 96

Enseñanza del derecho internacional humanitario en los Países Bajos . . . . . . . . . . . . . . 97

A propósito del derecho de asilo . . . . . . . 98 
REVUE INTERNATIONALE DE LA CROIX-ROUGE

INTERNATIONAL

REVIEW

OF THE RED CROSS

ARTICULOS Y PASAJES

DE LA REVISTA

EN LENGUA ALEMANA

Aparecen mensualmente ediciones en lenguas inglesa y francesa que pueden obtenerse en las mismas condiciones.

Das Henry-Dunant-Institut seit zehn Jahren - Teilnahme an den Genfer Abkommen - Falsche IKRKReiseausweise in Thailand ausgestellt - Hilfe für Saharaui-Flüchtlinge - Verbreitung der Genfer Abkommen

Publicada mensualmente por el Comité Internacional de la Cruz Roja.

17, avenue de la Paix, 1211 Ginebra (Suiza) - Número de cuenta postal 12-1767.

Subscripción anual: 30.- Fr.; cada número: 3.- Fr.

REDACCIÓN: J.-G. LOSSIER.

El Comité Internacional de la Cruz Roja sólo se responsabiliza de los textos publicados con su firma. 